\title{
Beyond Graphene, Chemistry of Graphene Derivates and Its Inorganic Analogues
}

\author{
Zdeněk Sofer \\ Department of Inorganic Chemistry \\ University of Chemistry and Technology Prague \\ Technická 5, 166 28, Prague 6 \\ Czech Republic
}

The chemistry of graphene is growing rapidly in last decade and broad range of graphene derivates was prepared. Nevertheless, only two stoichiometric derivates are currently known - hydrogenated and fluorinated graphene (graphane and fluorographene). Compared to graphene, these materials exhibit significantly different properties, e.g. higher reactivity as well as large differences in physical properties. Especially fluorographene can be applied as a substrate for nucleophilic substitution reactions, which significantly extend the possible chemical modifications of graphene. Derivatisation can be effectively performed by organometallic reagents like Grignard reagents, which can be effectively used for introduction of e.g. triple bond for click-chemistry reactions. The nucleophilic substitutions also enable the introduction of other functionalities, e.g. amines, thiols as well as hydrogen due to the higher difference in bond polarity. The possibility to introduce new moiety to these derivates is significantly higher in comparison with graphene oxides where the combination of various oxygen functionalities significantly reduces the scope of such modifications.

Currently, the chemistry of the other 2D materials starts to be explored. However, the chemistry of inorganic 2D materials like pnictogens and transition metal dichalcogenides is not well known and only several procedures were already reported. In comparison with graphene, new synthetic protocols have to be applied because the chemistry of these materials is extremely variable. In the case of transition metal dichalcogenides, the formation of the $\mathrm{M}-\mathrm{X}-\mathrm{C}$ bond ( $\mathrm{M}$ is a metal, $\mathrm{X}$ is any chalcogenide) can be used as a starting point for exploring their chemistry and for further derivatisation.

The chemistry of layered pnictogens is significantly different. In this case, various reactions including nucleophilic substitution can be applied, however, the bonding through the oxygen functionalities on pnictogen surface is observed in many cases. 